\title{
Guía clínica para el manejo de la faringoamigdalitis aguda del adulto
}

\author{
Josep Maria Cots Yago', Juan-Ignacio Alós Cortés², Mario Bárcena Caamaño ${ }^{3}$, Xavier Boleda Relats ${ }^{4}$, \\ José Luis Cañada Merino ${ }^{5}$, Niceto Gómez Gabaldón ${ }^{5}$, Ana Mendoza Barbero ${ }^{4}$, Isabel Vilaseca González ${ }^{6}$, \\ Carles Llor Vila ${ }^{1}$
}

1. Sociedad Española de Medicina de Familia y Comunitaria (SemFYC). 2. Grupo de Estudio de la Infección en Atención Primaria de la Sociedad Española de Enfermedades Infecciosas y Microbiología Clínica (GEIAP-SEIMC). 3. Sociedad Española de Médicos Generales y de Familia (SEMG). 4. Sociedad Española de Farmacia Familiar y Comunitaria (SEFAC). 5. Sociedad Española de Médicos de Atención Primaria (SEMERGEN). 6. Sociedad Española de Otorrinolaringología y Patología Cérvico-Facial (SEORL-PCF).

\section{PALABRAS CLAVE}

Faringoamigdalitis aguda, adulto,

diagnóstico, tratamiento. Streptococcus pyogenes, antibióticos

\section{ABREVIATURAS}

AEBHGA: estreptococo beta-hemolítico del grupo A.

FAA: farigoamigdalitis aguda.

NICE: National Institute for Health and Clinical

Excellence.

VIH: virus de la inmunodeficiencia humana.

\section{KEYWORDS}

Acute pharyngotonsillitis, adult, diagnosis, treatment. Streptococcus pyogenes, antibiotics

Recibido: 28/11/2014

Aceptado: 20/1/2015

Disponible online: 1-3-2015

\section{RESUMEN}

La faringoamigdalitis aguda (FAA) en el adulto es una de las enfermedades infecciosas más comunes en la consulta del médico de familia. La etiología más frecuente es viral. Dentro de la etiología bacteriana, el principal agente responsable es Streptococcus pyogenes o estreptococo $\beta$-hemolítico del grupo A (EBHGA), causante del 5-30\% de los casos. En el manejo diagnóstico las escalas de valoración clínica, para predecir la posible etiología bacteriana, son una buena ayuda para seleccionar a qué pacientes se deben practicar las técnicas de detección rápida de antígeno estreptocócico. Es conocido que, en general, sin estas técnicas, se tiende al sobrediagnóstico de FAA estreptocócica, con la consiguiente prescripción innecesaria de antibióticos, muchas veces de amplio espectro. Así, con el manejo de las escalas y la técnica de diagnóstico rápido, elaboramos los algoritmos de manejo de la FAA. Los objetivos del tratamiento son acelerar la resolución de los sintomas, reducir el tiempo de contagio y prevenir las complicaciones supurativas locales y no supurativas. Los antibióticos de elección para el tratamiento de la FAA estreptocócica son penicilina y amoxicilina. La asociación de amoxicilina y clavulánico no está indicada en el tratamiento inicial en la infección aguda. Los macrólidos tampoco son un tratamiento de primera elección; su uso debe reservarse para pacientes con alergia a la penicilina. Es importante en nuestro país adecuar tanto el diagnóstico de la FAA bacteriana y la prescripción de antibióticos a la evidencia científica disponible. La implantación de protocolos de actuación en las farmacias comunitarias puede ser de utilidad para identificar y cribar los casos que no requieran tratamiento antibiótico.

Clinical guide for the management of acute pharyngotonsillitis in adults

\section{ABSTRACT}

The acute pharyngotonsillitis (APT) in adults is one the most common infectious diseases in the family physician's surgery. The most frequent etiology is viral. Within the bacterial etiology, the main agent responsible is Streptococcus pyogenes or streptococcus $\beta$-GROUP A hemolytic (EBHGA), causing $5-30 \%$ of cases. In the diagnostic management, to predict the possible bacterial etiology, clinical evaluation scales are a good help for selecting which patients should undergo quick detection techniques for the streptococcic antigen. It is known that, in general, without these techniques streptococcic APT tends to be overdiagnosed, with the ensuing unnecessary prescription for antibiotics, often broad-spectrum. Thus, with the management of the steps and the quick diagnosis technique, we can draw up algorithms for managing APT. The objectives of the treatment are to accelerate the resolution of symptoms, reduce contagion time and prevent local suppurative and non-suppurative complications. The antibiotics of choice for treating streptococcic APT are penicillin and amoxicillin. The combination of amoxicillin and clavulanic acid is not indicated for the initial treatment of acute infection. Macrolides are not a first-choice treatment either; their use must be reserved for patients with allergy to penicillin. In our country it is important to adapt both the diagnosis of bacterial APT and the prescription of antibiotics to the scientific evidence available. The implementation of protocols of action in community pharmacies may be of use in identifying and screening cases that do not require antibiotic treatment.

Financiación: Ninguna ajena.

Conflicto de intereses: Los autores declaran no existir conflicto de intereses en relación con el contenido del artículo. Cite este artículo como: Cots JM, Alós Jl, Bárcena M, Boleda X, Cañada JL, Gómez N, Mendoza A, Vilaseca I, Llor C. Guía clínica para e manejo de la faringoamigdalitis aguda del adulto. Farmacéuticos Comunitarios. 2015 Mar 01; 7(1):20-31. doi:10.5672/FC.2173-9218. (2015/Nol7).001.04

Autora para correspondencia: Ana Mendoza (amendoza@sefac.org).

ISSN 1885-8619 @SEFAC (Sociedad Española de Farmacia Familiar y Comunitaria). Todos los derechos reservados. 


\section{Introducción}

La faringoamigdalitis aguda (FAA) es una de las infecciones respiratorias más frecuentes en nuestro medio. La FAA representa una causa no despreciable de absentismo laboral, de hasta 6,5 días de media de baja laboral por episodio $(1,2)$. Es también una de las razones más frecuentes por las que se prescribe un antibiótico en nuestro país, con una tasa aproximada de prescripción del 80\%. A pesar de ello, la causa más frecuente de etiología bacteriana, la producida por Streptoccus pyogenes o estreptococo $\beta$-hemolítico del grupo A (EBHGA) causa el 20\%$30 \%$ de todas las faringoamigdalitis en niños y el 5-15\% en adultos (2,3).

Uno de los principales problemas a los que se enfrenta el médico de Atención Primaria ante la FAA es el de poder realizar un diagnóstico diferencial etiológico de sospecha en función del cual poder instaurar el tratamiento más adecuado. La prescripción antibiótica ante una FAA es, en general, exagerada ya que la mayoría de los casos obedece a una causa viral. El uso excesivo de antibióticos conlleva la posibilidad de producir efectos secundarios en el paciente, la selección de resistencias y el consiguiente aumento en el gasto sanitario (4-7).

En la práctica habitual, el diagnóstico se realiza en base a criterios clínicos (fiebre, exudado amigdalar, adenopatia cervical anterior y ausencia de tos), que tienen una baja sensibilidad para predecir infeccion por EBHGA (49-74\%), por lo que la indicación de la prescripción antibiótica aumenta al haber un gran número de falsos positivos (8).

Para el diagnóstico de la FAA la prueba de referencia es el cultivo de exudado amigdalar, cuya sensibilidad y especificidad son muy elevadas (9095\% y >95\% respectivamente) (9). El periodo de tiempo necesario para realizar la lectura del cultivo constituye la principal limitación para su uso diagnóstico habitual. Es por ello que se han desarrollado técnicas inmunológicas rápidas de sencilla utilización $\mathrm{y}$ de bajo coste, que permiten en unos minutos poder detectar antígeno estreptocócico (10). La mayoria de estos tests tiene actualmente una alta especificidad (>95\%), pero su sensibilidad es de aproximadamente el $80 \%$, con un rango que oscila entre el 60\% y el 98\%, aunque varía según marcas comerciales y según síntomas y sig- nos del paciente (11). En cuanto al tratamiento el EBHGA continua siendo $100 \%$ sensible a penicilina y éste debe seguir siendo el tratamiento de elección (12).

\section{Etiología}

Varios virus y bacterias pueden causar FAA en el paciente inmunocompetente. Entre los virus, los adenovirus son de los más prevalentes. Otros virus implicados son rinovirus, enterovirus, virus influenza A y B, virus parainfluenza, virus respiratorio sincitial, coronavirus, metapneumovirus humano, virus de Epstein-Barr, virus del herpes simple, citomegalovirus y el virus de la inmunodeficiencia humana tipo 1 (VIH-1).

Las causas bacterianas más frecuentes son EBHGA, que causa hasta el 30\% de los casos en población infantil, pero es menos frecuente en los adultos. Es habitual la existencia de portadores asintomáticos, principalmente entre los niños. Otras bacterias implicadas en la FAA en nuestro medio son Streptococcus dysgalactiae subsp, equisimilis (estreptococos $\beta$-hemolíticos de los grupos C y G). Más raramente, la FAA puede estar causada por Fusobacterium necrophorum, Borrelia vincentii, Arcanobacterium haemolyticum, Neisseria gonorrhoeae (en adolescentes y adultos que practican sexo oral-genital), Mycoplasma pneumoniae (causa además bronquitis aguda o infección respiratoria superior) y Chlamydophila pneumoniae $(13,14)$.

Los estreptococos causantes de FAA mantienen hasta la fecha sensibilidad a las penicilinas y a otros antibióticos $\beta$-lactámicos, a pesar del uso masivo de éstos. No se ha descrito ninguna cepa resistente a la penicilina y las concentraciones mínimas inhibitorias de penicilina $G$ no han variado significativamente en los últimos 90 años. Los macrólidos y las lincosamidas (clindamicina) son considerados el tratamiento de elección en pacientes alérgicos o con sospecha de alergia a $\beta$-lactámicos. En los últimos años la resistencia a esos grupos ha aumentado en distintas partes del mundo, incluyendo España. Sin embargo, este problema no afecta de la misma manera a todos; mientras que los macrólidos de 14 átomos (eritromicina, claritromicina) y 15 átomos (azitromicina) presentan cifras de re- sistencia del 10-30\%, los de 16 átomos (midecamicina, josamicina) y las lincosamidas se mantienen por debajo del 7\% de resistencia (15). En cada zona debe conocerse y actualizarse la prevalencia de resistencia a macrólidos-lincosamidas, para tener disponibles alternativas a las penicilinas.

\section{Epidemiología}

La infección aguda de la mucosa y tejido orofaringeo constituye una de las causas principales de las consultas en Atención Primaria (50\% de los motivos de consulta por infección respiratoria alta) e incluso en los servicios de urgencias tanto hospitalarios como extrahospitalarios. Muchas de ellas tienen un carácter autolimitado y el uso de antibióticos en estos casos no estaría indicado.

En la práctica médica diaria preocupa de forma especial aquellas que se encuentran producidas por el EBHGA $(1,2)$. Es muy poco frecuente antes de los 3 años, tiene un pico de máxima incidencia entre los 5 y 15 años, para descender posteriormente entre un 5 y un 23\% en los adultos jóvenes $\mathrm{y}$ ser finalmente muy poco frecuente en mayores de 50 años (16).

En lo referente a su presentación, la mayor incidencia de todas ellas suele ser en las estaciones de invierno y primavera. Existen algunas excepciones como podrían ser las causadas por rinovirus $u$ otros virus que producen infecciones respiratorias de vías altas que predominan durante el final de la primavera o incluso al principio del verano como ocurre con las causadas por las del grupo de los adenovirus.

El mecanismo de transmisión suele producirse por vía respiratoria a través de las pequeñas gotas de saliva que se expelen al toser, estornudar o simplemente hablar desde una persona infectada a un huésped susceptible. También en algunos casos se han descrito brotes transmitidos por contaminación de alimentos o el agua, como también es posible su propagación a través de las manos. Es posible contagiarse una faringoamigdalitis estreptocócica a través de tocar las llagas de las infecciones por EBHGA en la piel. Por el contrario, la transmisión por fómites no parece desempeñar un papel importante en la transmisión de estos microorganismos causantes de la FAA $(17,18)$. 
Tabla 1 Diferencias clínicas entre faringoamigdalitis viral y bacteriana

\begin{tabular}{|l|l|l|}
\hline Caracteristicas & Viral & Bacteriana \\
\hline Edad & $<4$ años y $>45$ años & $5-15$ años \\
\hline Estacional & Variable & Invierno-primavera \\
\hline Inicio & Gradual & Brusco \\
\hline Síntomas & Fiebre leve Odinofagia leve & Fiebre elevada \\
& & Odinofagia importante \\
\hline Otros sintomas & Tos, conjuntivitis, rinitis, mialgias, diarrea & Cefalea, náuseas, vómitos, exantema \\
\hline Faringe & Eritematosa & Inflamación importante \\
\hline Adenopatias & Exudado (65\%) & Exudado (70\%) \\
\hline
\end{tabular}

Como factores de riesgo destacan los antecedentes familiares, las condiciones de hacinamiento familiar y la contaminación ambiental que incluye el tabaquismo crónico. Todos los grupos poblacionales están igualmente expuestos independientemente de su nivel socioeconómico o profesión.

En el caso de un paciente adulto, la incidencia de presentación es bastante más baja, pero también puede ser una causa frecuente de consulta en Atención Primaria, fundamentalmente aquellas que son de naturaleza viral. Si la causa es el EBHGA suele condicionar absentismo laboral que puede alcanzar hasta los seis días de baja por cada episodio.

\section{Clínica}

La mayor parte de las FAA son de origen viral y ocurren en el contexto de un cuadro catarral. Suelen presentarse en forma de brotes epidémicos y se acompañan de síntomas virales como la congestión nasal, febrícula, tos, disfonía, cefalea, o mialgias. La FAA bacteriana cursa con un cuadro brusco de fiebre alta con escalofríos, odinofagia y disfagia importantes, pero sin síntomas virales generales. En la tabla 1 se reflejan las principales diferencias clínicas entre la etiología viral y bacteriana. En la tabla 2 se describen los principales síntomas $\mathrm{y}$ signos que sugieren una etiología especifica en la FAA.

Clásicamente, se han clasificado las FAA de origen viral como FAA rojas y las bacterianas como FAA blancas, en base a la presencia o no de exudado. Sin embargo, los hallazgos clínicos muchas veces se superponen (8). Así, hasta un 65\% de FAA viral cursan con exudado faríngeo y un
30\% de las bacterianas pueden cursar sin exudado.

\section{Complicaciones}

de la faringoamigdalitis aguda Complicaciones supurativas

Ocurren por afectación de las estructuras contiguas a la infección, o por extensión de la infección a las zonas de drenaje. De entre ellas destacan el flemón y el absceso periamigdalino, el absceso retrofaríngeo, la otitis media aguda, la sinusitis, la mastoiditis y la adenitis cervical supurativa. Más excepcionales son la tromboflebitis de la vena yugular interna (síndrome de Lemierre), la fascitis necrotizante, la meningitis o los abscesos metastásicos por diseminación hemática.

Las complicaciones supurativas pueden aparecer en un 1-2\% de las FAA bacterianas sin tratamiento o tratadas con un antibiótico inadecuado o mal cumplimentado (18). Diversos estudios publicados en los últimos tres años ponen de manifiesto que otros gérmenes distintos del EBHGA pueden causar estas complicaciones mucho más frecuentemente que el EBHGA, como por ejemplo, el causado por $S$. anginosus (19). También se discute actualmente sobre el posible rol de Fusobacterium en estas complicaciones (20).

Algunas publicaciones han sugerido que el descenso en la prescripción de antibióticos para infecciones respiratorias altas podría estar asociado a un incremento de las complicaciones. Sin embargo, estos estudios no han podido determinar si este incremento era más frecuente en el grupo no tratado que en el grupo tratado (21-23). Petersen et al. describieron que con el uso de antibióticos el riesgo de complicaciones supuradas durante el primer mes tras el diagnóstico disminuía significativamente, siendo el número de episodios necesarios que deberían tratarse para prevenir una complicación seria de más de 4.000 casos (24). Little et al. analizaron prospectivamente los factores predictivos de complicaciones supurativas tras FAA (25). Las complicaciones incluían amigdalitis, absceso periamigdalino, otitis media, sinusitis, impétigo, así como la necesidad de nueva consulta por falta de resolución de síntomas durante el mes siguiente al diagnóstico. La presencia de complicaciones supurativas fue del 1,3\% de los pacientes, con independencia de si habían sido tratados con antibióticos de forma inmediata, por prescripción diferida, o no habían sido tratados. Sin embargo, dos terceras partes de las complicaciones se presentaron con mayor frecuencia en pacientes con 0 a 2 criterios de Centor (25). Un 14,2\% requirieron una nueva consulta. La inflamación amigdalar severa y el dolor ótico severo fueron factores independientes de riesgo de complicación.

Desde el punto de vista clínico, deberiamos sospechar la posibilidad de una complicación cuando la evolución clínica no sigue un curso satisfactorio. La aparición de dolor intenso de predominio unilateral, con disfagia y trismus de intensidad variable debe hacer pensar en una celulitis o absceso periamigdalino. La exploración muestra en estos casos un abombamiento del paladar blando y desplazamiento de la amígdala hacia la línea media. La infección en estos casos suele ser polimicrobiana y requerirá en la mayoría de pacientes el drenaje quirúrgico. Excepcionalmente el absceso puede extenderse a los 
Tabla 2 Caracteristicas clínicas especificas en base al germen etiológico de la faringoamigdalitis aguda

\begin{tabular}{|c|c|}
\hline Germen & Características clínicas \\
\hline \multicolumn{2}{|l|}{ Virus } \\
\hline Rinovirus & Resfriado común. Predominio en otoño y primavera. \\
\hline Coronavirus & Resfriado común. Predominio en invierno. \\
\hline Influenza A y B & Resfriado común. \\
\hline Parainfluenza & Resfriado, crup laríngeo. \\
\hline Adenovirus & Fiebre faringoconjuntival. Predominio en verano. \\
\hline Virus Coxsackie A & $\begin{array}{l}\text { Suele afectar a niños. Brotes epidémicos en verano. } \\
\text { Fiebre alta. Odinofagia intensa. Hiperemia en pilares amigdalinos. Vesículas } \\
\text { pequeñas, superficiales, con halo rojo. Enfermedad boca-mano-pie. }\end{array}$ \\
\hline Virus del herpes simple 1 y 2 & $\begin{array}{l}\text { Gingivoestomatitis, vesículas y úlceras que afectan a faringe y cavidad oral. } \\
\text { Puede cursar con exudado faríngeo. }\end{array}$ \\
\hline Virus de Epstein-Barr (VEB) & $\begin{array}{l}\text { Mononucleosis infecciosa. Más frecuente en adolescentes. } \\
\text { Fiebre. Malestar general. Astenia. Mialgias. } \\
\text { Importante inflamación faringoamigdalar que puede ser obstructiva y requerir } \\
\text { tratamiento antiinflamatorio intenso. } \\
\text { Exudado amigdalar en } 50 \% \text { de los casos. } \\
\text { Inflamación ganglios cervicales. Esplenomegalia. Alteración hepática. } \\
\text { La toma de antibióticos puede desencadenar un exantema máculo-papular en } \\
\text { tórax y extremidades. }\end{array}$ \\
\hline Citomegalovirus & $\begin{array}{l}\text { Sindrome mononucleósico. Respecto a VEB menos intensa la faringoamigdalitis y } \\
\text { mayor aumento de transaminasas. }\end{array}$ \\
\hline HIV & $\begin{array}{l}\text { Primoinfección: Fiebre. Mialgias. Artralgias. } \\
\text { Exantema cutáneo. Linfadenopatías y ulceraciones mucosas sin exudado. }\end{array}$ \\
\hline \multicolumn{2}{|l|}{ Bacterias } \\
\hline Estreptococo Grupo A & $\begin{array}{l}\text { Faringoamigdalitis. } \\
\text { Escarlatina (por cepas productoras de toxinas eritrogénicas). Exantema máculo- } \\
\text { papuloso más acentuado en pliegues. Lengua aframbuesada. } \\
\text { Descamación durante convalecencia. } \\
\text { Posibilidad de fiebre reumática. }\end{array}$ \\
\hline Estreptococos grupos C y G & Faringoamigdalitis. \\
\hline Arcanobacterium haemolyticum & Faringoamigdalitis. Exantema escarlatiniforme. \\
\hline Neisseria gonorrhoeae & Faringoamigdalitis. \\
\hline Corynebacterium diphteriae & Exudados faringeos. Estridor. Alteraciones cardíacas. \\
\hline Anaerobios & Angina de Plaut-Vincent. Gingivoestomatitis. \\
\hline Fusobacterium necrophorum & $\begin{array}{l}\text { Tromboflebitis séptica de la yugular interna: dolor intenso, disfagia, tumefacción } \\
\text { y rigidez cervical. }\end{array}$ \\
\hline Francisella tularensis & $\begin{array}{l}\text { Faringoamigdalitis. Con antecedentes de consumo de carne silvestre poco } \\
\text { cocinada. }\end{array}$ \\
\hline Yersinia enterocolitica & Faringoamigdalitis. Enterocolitis. Puede cursar con exudados. \\
\hline Mycoplasma pneumoniae & Bronquitis. Neumonía. \\
\hline \multicolumn{2}{|l|}{ Hongos } \\
\hline Candida spp. & $\begin{array}{l}\text { Pacientes inmunodeprimidos, con múltiples tratamientos antibióticos, corticoides } \\
\text { inhalados o quimioradioterapia. Exudado blancuzco en faringe y cavidad oral. } \\
\text { Afectación superficial, sin fiebre ni adenitis. }\end{array}$ \\
\hline
\end{tabular}


espacios cervicales profundos y más raramente originar una fascitis necrotizante (26) o una tromboflebitis de la vena yugular interna.

\section{Complicaciones no supurativas}

Destaca la fiebre reumática aguda y la glomerulonefritis post-estreptocócica, que ocurre tras un periodo de latencia de unas semanas. La fiebre reumática es muy poco frecuente en los países desarrollados, con una incidencia anual inferior a 1 caso por cada 100.000 habitantes (18), pero se mantiene como la principal causa de enfermedad cardíaca adquirida en niños de países en desarrollo (14).

\section{Diagnóstico}

Con la evidencia disponible en la actualidad, el médico de Atención Primaria debe identificar principalmente la infección por EBHGA, ya que estos casos deben tratarse con antibióticos.

\section{Manifestaciones clínicas}

El diagnóstico acostumbra a ser clínico en nuestro país. Los hallazgos clínicos que suelen acompañar a la FAA causada por EBHGA son dolor de garganta, a menudo con aparición brusca, fiebre, dolor de cabeza, náuseas, vómitos y dolor abdominal, inflamación y/o presencia de exudado amigdalar y adenopatías cervicales dolorosas, sin tos. Sin embargo, ninguno de estos signos y síntomas es específico de la FAA por EBHGA, ya que los criterios clínicos tienen poca validez para discernir la causa estreptocócica del resto de causas.

\section{Escalas de predicción}

Diversos estudios han evaluado escalas de predicción clínicas que aumentan las probabilidades de infección causada por EBHGA. La más conocida es la de Centor, que usa cuatro criterios: fiebre, exudado o hipertrofia faringoamigdalar, adenopatías laterocervicales dolorosas y ausencia de tos, en la que se suma un punto por cada uno de los criterios presentes, oscilando la puntuación global de 0 a 4 (27). Se han elaborado otras escalas pronósticas, como las de McIsaac y, últimamente, la FeverPAIN creada por investigadores británicos. No obstante, la más utiliza- da y sencilla a la vez es la de Centor y es la que debe recomendarse.

Los pacientes con ninguno 0 sólo uno de estos criterios presentan un riesgo muy bajo de infección por EBHGA y, por tanto, no necesitan de ningún abordaje ni diagnóstico ni terapéutico. Las guías de práctica clínica más influyentes, tales como la de la Infectious Diseases Society of America o la del National Institute for Health and Clinical Excellence (NICE), así lo recomiendan $(11,28)$.

La mayoría de expertos opinan que no pueden usarse estas escalas de puntuación clínica sin una evaluación adicional para el diagnóstico de la FAA por EBHGA porque los médicos suelen sobreestimar la probabilidad de infección por esta causa (11). Esto está apoyado por dos estudios que mostraron que los pacientes con cuatro criterios presentan entre el 39 y el 57\% de probabilidades de presentar un cultivo faríngeo positivo para EBHGA (tabla 3). Los porcentajes más altos se hallan en estudios efectuados en niños de 5 a 14 años y los más bajos, en mayores de 15 años. Por tanto, el uso de estas escalas no es suficiente para conocer con exactitud si un paciente presenta una FAA causada por EBHGA o no. De hecho, la sensibilidad del juicio clínico oscila entre el 49 y 74\% y la especificidad entre el 58 y 76\% (8). Además, los médicos de Atención Primaria evalúan los distintos criterios de Centor de manera diferente; así, en España, los médicos dan mucho más peso al exudado faringoamigdalar en una FAA, ya que prescriben antibióticos 28 veces más si este signo está presente que a los otros 3 criterios (menos de 5 veces más si estos criterios se presentan) (29).

\section{Métodos microbiológicos}

El cultivo faríngeo es la prueba de referencia para conocer la etiología de la infección. Su principal desventaja es el tiempo que se tarda en obtener resultados. Al mismo tiempo, es muy probable que la etiología por anaerobios se haya infraestimado hasta ahora ya que su identificación requiere de unas condiciones de cultivo anaerobias estrictas que muchos laboratorios de microbiología no disponen. En los años 80 se desarrollaron pruebas de detección antigénica rápida del EBHGA (Strep A), en muestras faríngeas tomadas con torunda.
Estas técnicas presentan la ventaja de la disponibilidad del resultado en el mismo momento de la consulta. Se basan en la extracción del antígeno de carbohidratos del EBHGA a partir de los microorganismos obtenidos del exudado faríngeo. Son de sencilla aplicación en la consulta médica, debiéndose recoger la muestra con la ayuda de un depresor, inmovilizando la lengua y se realiza la toma del área amigdalar y faringe posterior, así como de cualquier zona inflamada o ulcerada. Es fundamental evitar rozar la torunda con la úvula, mucosa bucal, labios o lengua, tanto antes como después de la toma (30). Los hisopos son depositados en cubetas a las que se añade un reactivo que contiene anticuerpos antiestreptocócicos.

La validez depende de la técnica de recogida de la muestra (pueden darse resultados falsos negativos cuando se obtiene poco material), área donde se recoge (ofrece mejor rendimiento cuando se recoge de amígdalas y/o pared posterior de la faringe), procedimiento y condiciones del cultivo, probabilidad de infección estreptocócica (algunos autores han comprobado un sesgo de espectro, de forma que la sensibilidad del Strep A aumenta cuanto mayor es el número de criterios de Centor que presenta un paciente), presencia de otros gérmenes en la faringe (pueden darse resultados falsos positivos si la faringe presenta un crecimiento importante

Tabla 3 Criterios de Centory probabilidad de infección por estreptococo $\beta$-hemolítico del grupo A

\begin{tabular}{|l|l|}
\hline \multicolumn{2}{|l|}{ Criterios de Centor } \\
\hline Fiebre o historia de fiebre $>38^{\circ} \mathrm{C}$ \\
\hline Exudado o hipertrofia amigdalar \\
\hline Adenopatias laterocervicales dolorosas \\
\hline Ausencia de tos \\
\hline $\begin{array}{l}\text { Número de } \\
\text { criterios } \\
\text { de Centor }\end{array}$ & $\begin{array}{c}\text { Probabilidad de } \\
\text { infección por EBHGA }\end{array}$ \\
\hline Cuatro & $39-57 \%$ \\
\hline Tres & $25-35 \%$ \\
\hline Dos & $10-17 \%$ \\
\hline Uno & $<10 \%$ \\
\hline Cero & $<2,5 \%$ \\
\hline
\end{tabular}

EBHGA: Estreptococo $\beta$-hemolitico del grupo A. 
de Staphylococcus aureus), uso de pruebas más allá de la fecha de caducidad y marca comercial. Otro aspecto a considerar es que la positividad del Strep A no distingue infección aguda de estado de portador, aspecto que tampoco hace el cultivo. Los porcentajes de portadores asintomáticos puede llegar al 20\%, pero su prevalencia en adultos no llega al 5\% (31).

Se ha visto que los médicos que usan pruebas de detección antigénica rápidas prescriben menos antibióticos en la FAA que aquellos que no las utilizan (32). Sin embargo, aunque el valor predictivo negativo es muy elevado, un ensayo clínico reciente demostró que los médicos españoles prescribieron antibióticos en un poco más del 30\% de los casos con Strep A negativo (30). Esto puede ser debido a la costumbre de prescribir sistemáticamente antibióticos en pacientes que presentan al menos dos criterios de Centor.

Las pruebas de detección antigénica rápida empleadas para el diagnóstico etiológico de la FAA son específicas para el EBHGA y no descartan otras etiologías, como las producidas por $S$. dysgalactiae y $S$. angi- nosus, cuyas manifestaciones clínicas son similares. Estas pruebas ofrecen la ventaja de diagnosticar la FAA estreptocócica en unos pocos minutos, con una especificidad asociada mayor del 95\% cuando se utilizan en pacientes con dos o más criterios de Centor (11). No obstante, aparte de que estas pruebas no sirven para descartar otras causas distintas del EBHGA, en un estudio reciente se observó que tampoco evitan la aparición de complicaciones cuando sus resultados son falsos negativos (33). Por todo ello, es necesario seguir investigando nuevas pruebas de diagnóstico rápido más fiables que ayuden al médico de Atención Primaria a poder tomar de forma más clara la decisión de tratar o no tratar la FAA con antibióti$\cos (34)$.

\section{Diagnóstico recomendado}

La prueba de diagnóstico rápido a utilizar actualmente en Atención Primaria es el Strep A, su uso se recomienda únicamente en los casos en que se sospeche de una probable infección estreptocócica.
En los pacientes con ningún o un criterio de Centor las recomendaciones de los expertos y las guías de práctica clínica coinciden en que no hay que utilizar ningún test ni tratar con antibióticos.

En pacientes con dos criterios de Centor la situación es indeterminada y en la actualidad la guía NICE considera en este grupo la prescripción diferida de antibióticos (28). En un estudio publicado recientemente, Little et al. observaron que los pacientes asignados a la prescripción diferida de antibióticos consumieron menos de la mitad de antibióticos y refrecuentaron un 40\% menos que aquellos que fueron tratados de forma inmediata con antibióticos (35).

La estrategia que ha demostrado un mejor cociente coste-efectividad es la de realizar pruebas antigénicas rápidas a los pacientes con mayor probabilidad de infección por EBHGA y tratar en base al resultado, los casos positivos (36). Desde este punto de vista la mejor recomendación para los pacientes con dos o más criterios de Centor sería realizar técnicas rápidas de detección de antígenos (Strep A) (figura 1).

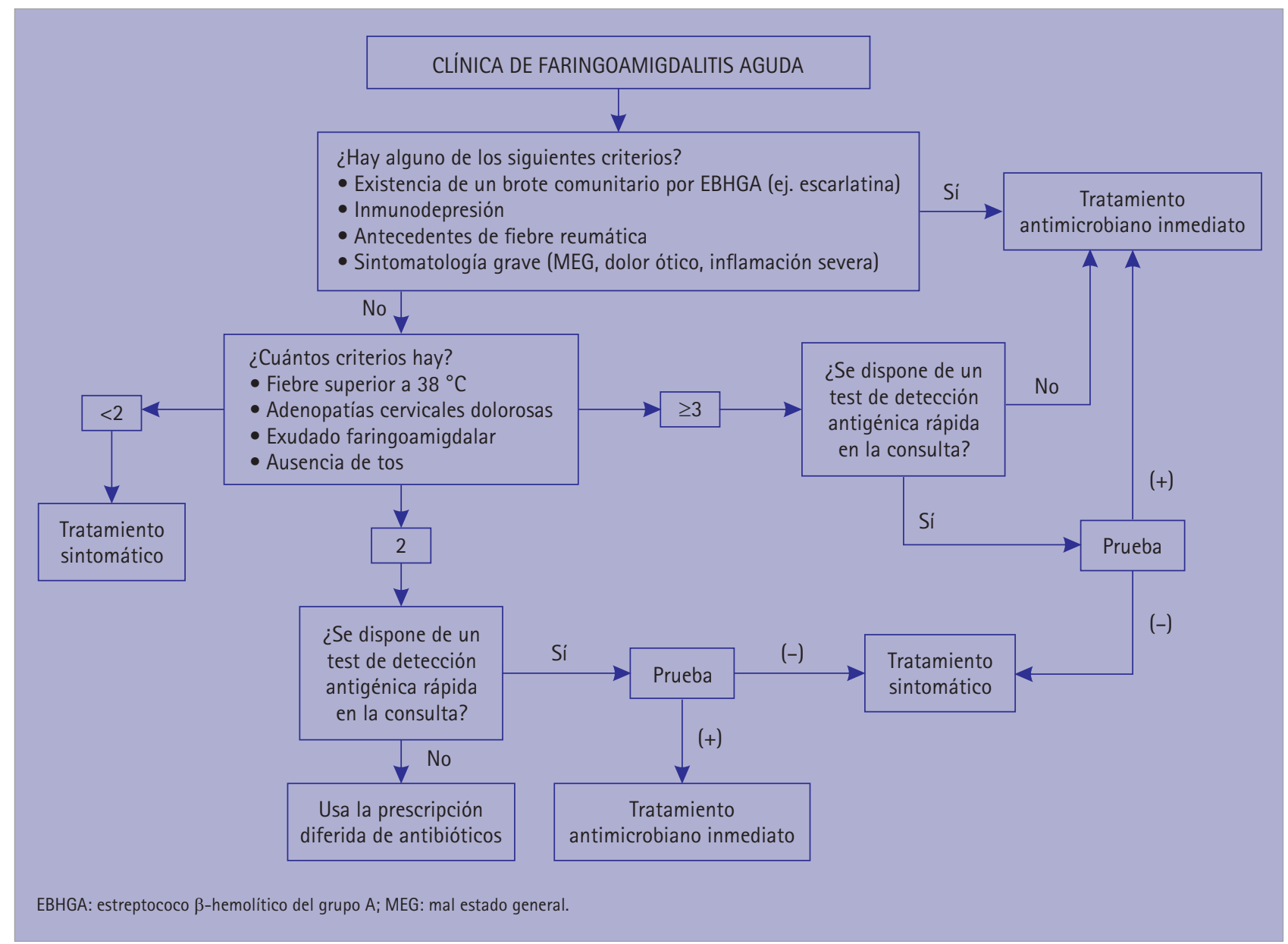

Figura 1 Abordaje recomendado de la faringoamigdalitis aguda en el adulto 
Esta recomendación coincide con la última propuesta de la Sociedad Española de Medicina Familiar y Comunitaria (37).

\section{Tratamiento}

Objetivos del tratamiento

Son objetivos de tratamiento antibiótico en la faringoamigdalitis por EBHGA:

- Acortar el curso de la enfermedad. El tratamiento antibiótico ha demostrado ser efectivo en reducir, aunque de forma muy marginal, la duración de la sintomatología de la faringoamigdalitis causada por EBHGA, concretamente en 16 horas (38). Esta diferencia es mayor en adolescentes y adultos jóvenes, puesto que el tratamiento antimicrobiano puede reducir en dos días la sintomatología en estos grupos.

- Erradicar el germen. Es importante en Atención Primaria identificar la FAA causada por EBHGA, ya que los pacientes que la presentan se benefician del tratamiento antimicrobiano.

- Evitar el contagio. El tratamiento antibiótico logra negativizar el cultivo en las primeras 24 horas en el 97\% de casos, disminuyendo el riesgo de contagio a otras personas.

- Prevenir las complicaciones. En algunos estudios el tratamiento antibiótico de la FAA causada por EBHGA ha disminuido la incidencia de complicaciones supurativas y no supurativas agudas como la fiebre reumática, aunque este efecto no se ha observado en todas las publicaciones.

- Mejorar los síntomas. En el paciente con FAA debemos utilizar fármacos para reducir la sintomatología principal que es el dolor de garganta, con la correcta prescripción de antinflamatorios y/o analgésicos.

En los últimos años se están publicando artículos sobre posibles beneficios de tratar con antibióticos otras causas de FAA (20). Existe discusión sobre la necesidad de tratar la infección causada por otros estreptococos -hemolíticos, principalmente los grupos C y G. El tratamiento antibiótico de la FAA por el estreptococo del grupo C se podría asociar a una ligera menor duración de síntomas, pero sólo en adultos (un día menos) (39). Se ha comprobado también que el estreptococo $\beta$-hemolítico del grupo $C$ puede causar glomerulonefritis y puede incluso causar algunos casos de fiebre reumática aguda. Más dudas existen sobre el beneficio del tratamiento antibiótico en la FAA causada por el estreptococo del grupo G. Otra causa que ha merecido en los últimos años mucha atención es la infección por Fusobacterium necrophorum. Sin embargo, no hay certeza de que la antibioterapia pueda disminuir la duración de la sintomatología de la FAA causada por este anaerobio. Como se ha comentado anteriormente, la necesidad de tratar la etiología por $S$. del grupo anginosus tampoco está clara (19).

\section{Tratamiento antibiótico}

Debe administrarse el tratamiento antibacteriano durante al menos 8 dias, aunque preferentemente se recomienda administrarlo durante 10 días ya que la mayoría de estudios se han efectuado con esta duración. En caso de positividad del Strep A debe recomendarse la utilización de fenoximetilpenicilina o penicilina V (1.200.000 $\mathrm{UI} / 12$ horas por vía oral), ya que el EBHGA ha sido y sigue siendo sensible a este antibiótico en todo el mundo (12).

En caso de intolerancia al tratamiento de elección puede administrarse amoxicilina $500 \mathrm{mg} / 12$ horas
(40). También puede administrarse una cefalosporina de primera generación, como cefadroxilo $500 \mathrm{mg} / 12$ horas (40). Si hay alergia confirmada a la penicilina se aconseja utilizar clindamicina $300 \mathrm{mg} / 8$ horas durante 10 días o un macrólido de 16 átomos como josamicina, $1 \mathrm{~g} / 12$ horas durante 10 días puesto que las resistencias del EBHGA, aunque han disminuido en los últimos años, siguen siendo mayores frente a los macrólidos de 14 y 15 átomos que a los de 16 átomos. En caso de FAA estreptocócica de repetición se puede administrar la asociación de amoxicilina y ácido clavulánico 500/125 mg/8 horas durante 10 días (tabla 4).

\section{Tratamiento sintomático}

Se recomienda reposo durante el proceso febril, la toma adecuada de líquidos, evitar irritantes y gárgaras con agua caliente y sal (41).

Como tratamiento farmacológico no antibiótico, la reciente guía europea sobre manejo de la FAA recomienda el uso de analgésicos y antiinflamatorios (14). Ibuprofeno y diclofenaco son ligeramente más efectivos que paracetamol para el alivio del dolor de garganta. Flurbiprofeno, antinflamatorio de acción local, ha demostrado ser más eficaz que el placebo para disminuir el dolor de garganta $(42,43)$. En este sentido, el uso de antiinflamatorios de acción local puede ser una alternativa

Tabla 4 Tratamiento especifico de la faringoamigdalitis aguda por estreptococo $\beta$-hemolítico del grupo $A$

\begin{tabular}{|l|l|l|}
\hline Antibiótico & Dosis & Duración \\
\hline Primera elección & & \\
\hline Penicilina V (fenoximetilpenicilina) & $1,2 \mathrm{M} \mathrm{de} \mathrm{Ul/oral} / 12 \mathrm{~h}$ & $8-10$ días \\
\hline Alternativas & & \\
\hline Penicilina G & $1,2 \mathrm{M} \mathrm{de} \mathrm{Ul} \mathrm{im}$ & 1 dosis \\
\hline Amoxicilina & $500 \mathrm{mg} / 12 \mathrm{~h}$ & $8-10$ días \\
\hline Cefadroxilo & $500 \mathrm{mg} / 12 \mathrm{~h}$ & $8-10$ dias \\
\hline Alérgicos a $\beta$-lactámicos & & \\
\hline Josamicina & $1 \mathrm{~g} / 12 \mathrm{~h}$ & 10 dias \\
\hline Diacetil midecamicina & $600 \mathrm{mg} / 12 \mathrm{~h}$ & 10 dias \\
\hline Antibióticos en recurrencias & & 10 dias \\
\hline Clindamicina & $300 \mathrm{mg} / 8 \mathrm{~h}$ & 10 dias \\
\hline Amoxicilina y ácido clavulánico & $500-125 \mathrm{mg} / 8 \mathrm{~h}$ & \\
\hline
\end{tabular}


para el tratamiento de los síntomas de dolor de garganta sin fiebre alta. La evidencia sobre el beneficio de fitoterapia y acupuntura en la FAA es inconsistente (14). Más dudas hay sobre el beneficio de los corticoides orales. En una revisión de 8 ensayos clínicos controlados con placebo, en el que se incluyeron 743 pacientes se observó que una pauta corta de corticoides por vía oral o intramuscular fue más beneficiosa que el placebo para resolver el dolor de la FAA. Este beneficio fue mayor para los adultos, aquellos con mayor sintomatología y aquellos con FAA estreptocócica. No obstante, la calidad de los estudios fue pobre y la mayoría se llevó a cabo en servicios de urgencias.

Una variedad de agentes tópicos, administrados en forma de comprimidos, enjuagues o aerosoles, se han empleado para aliviar los síntomas de la FAA. Ambroxol $20 \mathrm{mg}$ ha mostrado una ligera disminución de síntomas en un metaanálisis publicado recientemente, pero la calidad de los cinco trabajos incluido era pobre (42). En una revisión de la Cochrane Library, el gluconato de zinc ha demostrado reducir ligeramente el dolor de garganta comparado con placebo, pero presentó más efectos secundarios, por lo que tampoco se recomienda en la FAA.

Algunos preparados contienen anestésicos tópicos como la lidocaína y la benzocaína que producen alivio del dolor rápido de forma precoz, aunque los trabajos realizados presentan poca calidad metodológica y se han utilizado dosis heterogéneas $(43,44)$. No hay evidencia sobre el uso de caramelos o la miel.

\section{Derivación}

Las FAA son diagnosticadas y tratadas mayoritariamente en el primer nivel asistencial (45). En determinadas ocasiones nos encontramos con la presencia de situaciones en las que hay que derivar al nivel hospitalario, por lo que se hace necesario definir en qué casos el médico de Atención Primaria optará por dicha derivación, con la finalidad de solucionar el problema del paciente, racionalizar los recursos de que se dispone y ser más eficiente (46).

Debemos diferenciar entre derivación urgente y diferida.

\section{Derivación urgente}

Aquellos casos en los que se precise ingreso, manipulaciones instrumentales inmediatas o cuando el proceso pueda comprometer la buena evolución del paciente $(47,48)$ :

1. Faringoamigdalitis aguda

- Casos de más de 2 semanas de duración, con mala evolución.

- Casos con gran componente inflamatorio que, a pesar del tratamiento, impide la deglución normal.

- Sospecha de faringoamigdalitis lingual con obstrucción de vía aéreo-digestiva.

- Faringoamigdalitis con sospecha de infiltración linfomatosa o de cualquier otro proceso neoplásico.

2. Complicaciones locorregionales (49)

- Adenitis que evoluciona a un adenoflemón.

- Flemones y abscesos periamigdalinos.

- Infecciones del espacio parafaríngeo.

- Infecciones de los espacios retrofaríngeos, con gran riesgo de evolucionar a mediastinitis.

- Tortícolis de gran intensidad, que pueda corresponder a una subluxación atlanto-axoidea (síndrome de Grisel).

3. Complicaciones a distancia

- Síndrome de Lemierre: tromboflebilits de la vena yugular interna (empeoramiento con escalofríos, fiebre alta, dolor y tumefacción cervical ipsilateral en el ángulo de la mandíbula y a lo largo del músculo esternocleidomastoideo $\mathrm{y}$ rigidez de nuca).

\section{Derivación diferida}

Cuando se precise el manejo en el nivel hospitalario. La mayoría de las veces será referente a la necesidad de una amigdalectomía. Las indicaciones para ésta dentro de los procesos infecciosos son las siguientes (50):

1. Amigdalitis de repetición

Amigdalitis de repetición o amigdalitis recurrentes: situaciones clínicas con las siguientes características:

- Siete episodios o más de amigdalitis aguda al año en el último año, o

- Cinco episodios al año en los últimos 2 años, o

- Tres episodios al año en los últimos 3 años, o
- Síntomas persistentes durante al menos 1 año.

Además cada episodio debe cumplir, al menos, uno de los siguientes criterios clínicos:

- Exudado purulento amigdalar.

- Fiebre $>38^{\circ} \mathrm{C}$.

- Linfoadenopatías cervicales anteriores dolorosas.

- Cultivo faríngeo positivo para EBHGA.

Estos criterios son los denominados mínimamente aceptables. No obstante, cada caso debe ser evaluado en particular sopesando factores como lo incapacitante del cuadro, las repercusiones sobre el paciente y su familia.

2. Absceso periamigdalino recurrente Se indicará cirugía ante dos casos consecutivos de absceso periamigdalino ipsilateral.

\section{Adenitis cervical recurrente} Definida como:

- Inflamación aguda de adenopatías cervicales múltiples.

- Fiebre $>38^{\circ} \mathrm{C}$ y malestar general.

- Más de 3 días de duración.

- Ausencia de infección respiratoria baja.

- Coexistencia de infección respiratoria alta o amigdalitis aguda.

Los criterios de frecuencia y las consideraciones a la hora de evaluar estos casos son las mismas que las descritas para la amigdalitis recurrente.

\section{Manejo en la farmacia comunitaria}

El dolor de garganta es motivo frecuente de consulta en el ámbito sanitario, muchas de ellas en las farmacias comunitarias (51). Las farmacias comunitarias son centros sanitarios accesibles a la población; por ello, el abordaje de esta patología debe realizarse de una manera protocolizada a fin de decidir si actuar desde la farmacia comunitaria o derivar al médico.

La indicación farmacéutica es el servicio profesional prestado ante una demanda de un paciente o usuario que llega a la farmacia sin saber qué medicamento ha de adquirir y solicita al farmacéutico el remedio 


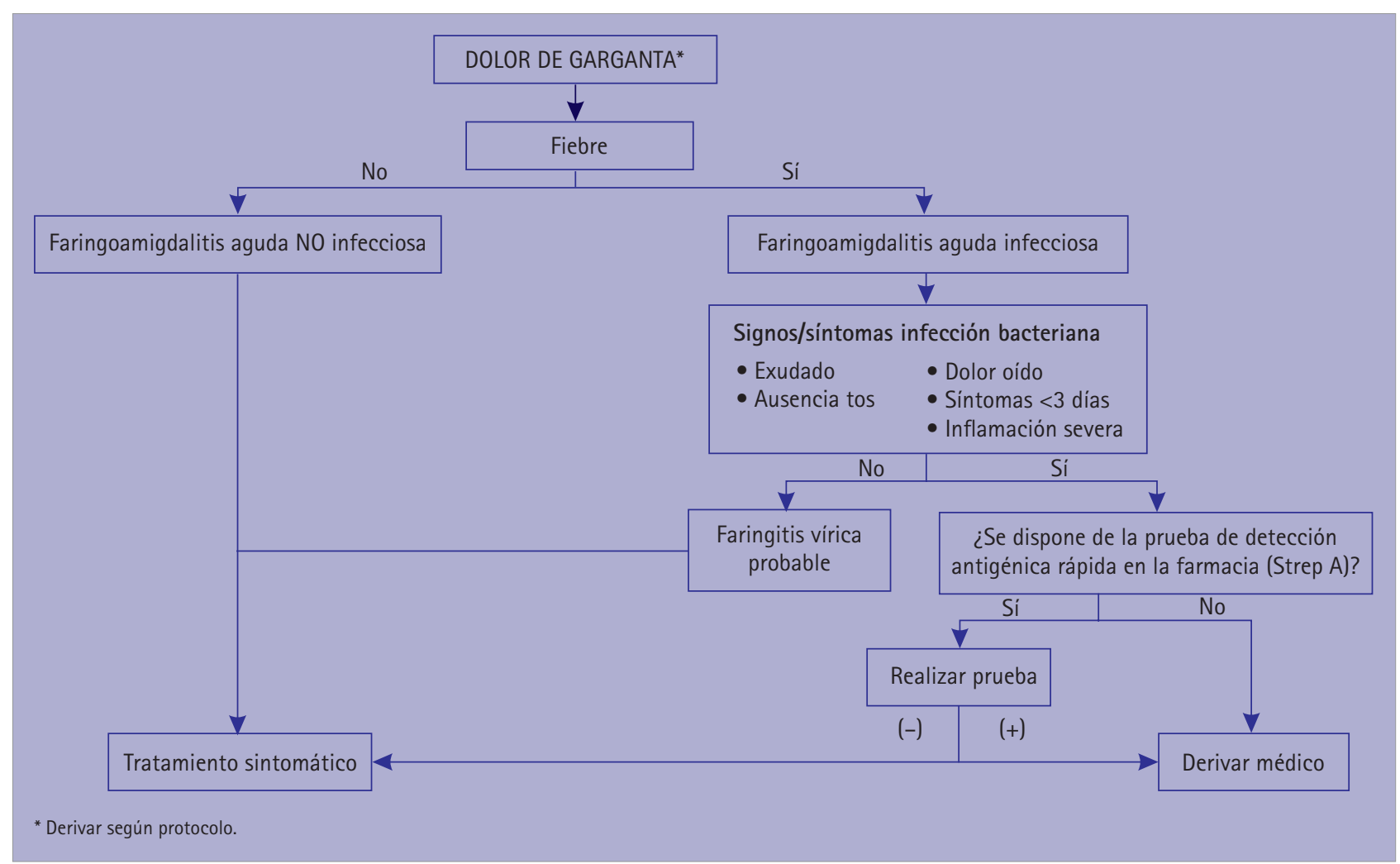

Figura 2 Manejo del dolor de garganta en la farmacia comunitaria

más adecuado para un problema de salud concreto. Si el servicio requiere la dispensación de un medicamento, se ha de realizar con criterios de atención farmacéutica, garantizando, después de una evaluación individual, que el paciente recibe y utiliza los medicamentos de forma adecuada a sus necesidades clinicas, en dosis precisas según sus requerimientos individuales, durante el periodo de tiempo adecuado, con la información para utilizarlos de manera correcta, y de acuerdo con la normativa vigente (52).

Dentro del procedimiento a seguir ante la consulta para solucionar el dolor de garganta, el farmacéutico debe considerar los siguientes puntos (figura 2):
- Quién realiza la consulta. Verificar si la persona que acude es la que tiene el problema de salud.

- Cuál es la razón de la consulta: dolor garganta.

- Se debe preguntar por los síntomas: en la farmacia comunitaria se tratan solamente problemas de salud autolimitados.

- Verificar

- Signos y síntomas. Si se cumplen criterios de etiología bacteriana, derivar al médico $u$ ofrecer realización de Strep A. Así la técnica está validada y el profesional farmacéutico esté correctamente formado para su realización (tabla 5).

- Si ha habido un tratamiento anterior sin obtener mejoría.
- Utilización de otros medicamentos para otras causas.

- Enfermedades concomitantes u hospitalización reciente.

- Presencia de alergias o intolerancias.

- Presencia de una situación fisiológica especial (embarazo...).

- Evaluar

- Criterios de derivación al médico (tabla 6).

- Contraindicaciones.

- Posibles interacciones de la medicación de base del paciente con el tratamiento que se vaya a recomendar.

- Actuar. Utilizando una o más opciones de las siguientes:

- Asesorar sin dispensar. Consejos higiénico-dietéticos:

Tabla 5 Uso de Strep A en la farmacia comunitaria

- Si se cumplen criterios de etiología bacteriana, el farmacéutico ofrecerá la realización de una prueba de Strep A si la técnica está validada y el profesional farmacéutico está correctamente formado para su realización.

- La realización de esta prueba diagnóstica en la farmacia comunitaria es útil para (47):

- Disuadir al paciente que solicita un tratamiento antibiótico sin prescripción médica.

- Hacer un cribado de la FAA de origen bacteriana.

- Facilitar al médico el diagnóstico de la enfermedad.

- En caso de resultado negativo se realizará la actuación farmacéutica correspondiente.

- En caso de resultado positivo, además del tratamiento sintomático, se le recomendará que acuda a su médico para confirmar el diagnóstico e instaure el tratamiento adecuado 
Tabla 6 Criterios de derivación al médico desde la farmacia comunitaria

\begin{tabular}{l} 
Criterios claros de derivación \\
\hline Repercusión sistémica, con fiebre elevada y mal estado general \\
Historia previa de fiebre reumática \\
Otros criterios de derivación \\
\hline Edad menor de 15 años \\
\hline Pacientes con enfermedad de base mal controlada (diabéticos, inmunodeprimidos...), \\
y/o situación fisiológica que lo requiera \\
\hline Pacientes que presenten disfonía de más de 3 semanas de evolución \\
\hline Presencia de paladar abombado o con voz nasal \\
Si el paciente ha tomado antibiótico hace menos de una semana sin mejoría \\
\hline
\end{tabular}

- Aumentar ingesta de líquidos.

- Dieta ligera.

- Utilizar pañuelos de papel de un solo uso.

- Lavarse las manos frecuentemente.

- Evitar fumar.

- Humedecer el ambiente, manteniendo una ventilación adecuada.

- No forzar la voz.

- Evitar cambios bruscos de temperatura.

- Dispensar un tratamiento farmacológico que no precise prescripción médica

- Analgésico-antiinflamatorio no esteroideo de acción local: flurbiprofeno.

- Analgésico-antiinflamatorio no esteroideo sistémico: ibuprofeno.

- Analgésicos: paracetamol.

- Recomendar tratamiento no farmacológico

- Suero fisiológico.

- Derivar al médico si es necesario.

- Seguimiento farmacoterapéutico para maximizar la efectividad y seguridad de los tratamientos, minimizando riesgos, contribuir a la racionalización de los medicamentos y mejorar la calidad de vida de los pacientes (53).

\section{Conclusiones}

El objetivo principal del documento de consenso es orientar el manejo de la FAA en atención primaria y en las farmacias comunitarias.

1. La etiología más frecuente de la FAA es la viral. Entre las causas bacterianas, el principal agente responsable es el EBHGA.
2. En general, dada la inespecificidad de los síntomas y signos clínicos, se tiende al sobrediagnóstico de FAA estreptocócica, con la consiguiente sobreprescripción innecesaria de antibióticos.

3. Para ayudar al diagnóstico etiológico son de utilidad las escalas de valoración clínica, que permiten seleccionar a quiénes se deben practicar un test de diagnóstico rápido.

4. Cuando disponemos de test de diagnóstico rápido debe utilizarse según unos criterios, no en todas

5. Se recomienda utilizar el test Strep A cuando el paciente presenta dos o más criterios en la escala de Centor.

6. El antibiótico de elección para el tratamiento de la FAA estreptocócica es la penicilina $\mathrm{V}$ o fenoximetilpenicilina.

7. La efectividad de la penicilina está demostrada y no se ha descrito, hasta el momento, ningún caso de EBHGA resistente. Su espectro de acción es reducido y por tanto selecciona menos resistencias que otros antibióticos.

8. La asociación de amoxicilina y ácido clavulánico no está indicada de forma empírica en el tratamiento de la FAA estreptocócica no recurrente. El EBHGA no produce $\beta$-lactamasas.

9. Es necesario en nuestro país adecuar la prescripción de antibióticos a la evidencia científica disponible.

10. La farmacia comunitaria, como servicio de atención sanitaria, debe manejar la FAA con la aplicación de protocolos para saber qué pacientes precisan atención farmacéutica y qué pacientes precisan atención médica. las FAA.

\section{Referencias bibliográficas}

1. Estudio Nacional de la Infección Respiratoria (ENIR). Gabinete de Estudios Sociológicos, SOCIMED Madrid 1990.

2. Bisno AL. Acute pharyngitis: etiology and diagnosis. Pediatrics 1996;97: 949-54.

3. Ebell MH, Smith MA, Barry HC, Ives $\mathrm{K}$, Carey $\mathrm{M}$. The rational clinical examination. Does this patient have strep throat? Jama 2000;284:2912-8. doi:10.1001/jama.284.22.2912

4. Goossens H, Ferech M, Vander Stichele R, Elseviers M, Group EP. Outpatient antibiotic use in Europe and association with resistance: a cross-national database study. Lancet 2005;365:579-87. doi:10.1016/ S0140-6736(05)17907-0

5. EDDC/EMEA Joint Technical Report. The bacterial challenge: time to react. A call to narrow the gap between multidrug-resistant bacteria in te EU and the development of new antibacterial agents. Stockholm. 2009.

6. Costelloe C, Metcalfe C, Lovering A, Mant D, Hay AD. Effect of antibiotic prescribing in primary care on antimicrobial resistance in individual patients: systematic review and meta-analysis. Bmj 2010;340:c2096. doi:10.1136/bmj.c2096

7. World Health Organization. WHO's first global report on antibiotic resistance - global surveillance report. Virtual Press Conference 2014.

8. Andersen JS, Borrild NJ, Hoffmann S. Antibiotics for sore throats. Potential of antigen detection tests. BMJ 1995;310:58-9. doi:10.1136/ bmj.310.6971.58c

9. Kellogg JA. Suitability of throat culture procedures for detection of group A streptococci and as reference standards for evaluation of streptococcal antigen detection kits. J Clin Microbiol 1990;28:165-9.

10. Llor C, Hernández Anadón S, Gomez Bertomeu FF, Santamaría Puig JM, Calvino Domínguez 0, Fernández Pages Y. [Validation of a rapid antigenic test in the diagnosis of pharyngitis caused by group a beta-haemolytic Streptococcus]. Atencion primaria / Sociedad Espanola de Medicina de Familia y Comunitaria 2008;40:489-94.

11. Shulman ST, Bisno AL, Clegg HW, Gerber MA, Kaplan EL, Lee G, et al. Clinical practice guideline for the diagnosis and management of group A streptococcal pharyngitis: 2012 update by the Infectious Diseases Society of America. Clinical infectious diseases: an official publication of the Infectious Diseases Society of America 2012;55:1279-82. doi:10.1093/cid/cis847

12. Ralph AP, Carapetis JR. Group a streptococcal diseases and their 
global burden. Curr Top Microbiol Immunol 2013;368:1-27. doi:10. 1007/82_2012_280

13. Wessels MR. Clinical practice. Streptococcal pharyngitis. N Engl J Med 2011;364:648-55. doi:10.1056/NEJMср1009126

14. Group ESTG, Pelucchi C, Grigoryan L, Galeone C, Esposito S, Huovinen P, et al. Guideline for the management of acute sore throat. Clinical microbiology and infection : the official publication of the European Society of Clinical Microbiology and Infectious Diseases 2012;18 Suppl 1:1-28.

15. Tamayo J, Perez-Trallero E, Gomez-Garces JL, Alos JI, Spanish Group for the Study of Infection in the Primary Health Care S. Resistance to macrolides, clindamycin and telithromycin in Streptococcus pyogenes isolated in Spain during 2004. The Journal of antimicrobial chemotherapy 2005;56:780-2. doi:10.1093/jac dki286

16. Cenjor C, García-Rodríguez JA, Ramos A, Cervera J, Tomás M, Asensi $\mathrm{F}$, et al. [Patient consent to "antimicrobial treatment of tonsillitis"]. Acta otorrinolaringologica espanola 2003;54:369-83. doi:10.1016/S00016519(03)78425-8

17. Sociedad Española de Quimioterapia; Sociedad Española de Otorrinolaringología y Patología Cérvico Facial; Sociedad Española de Infectología Pediátrica; Sociedad Española de Medicina General; Sociedad Española de Medicina Rural y Generalista; Sociedad Española de Medicina de Urgencias y Emergencias. Documento de consenso sobre tratamiento antimicrobiano en la faringoamigdalitis aguda. Rev Esp Quimioter 2003;15:74-88.

18. Pineiro Pérez R, Hijano Bandera F, Alvez González F, Fernández Landaluce A, Silva Rico JC, Pérez Canovas $\mathrm{C}$, et al. [Consensus document on the diagnosis and treatment of acute tonsillopharyngitis]. Anales de pediatría 2011;75:342 e1-13.

19. Hidaka H, Kuriyama S, Yano H, Tsuji I, Kobayashi T. Precipitating factors in the pathogenesis of peritonsillar abscess and bacteriological significance of the Streptococcus milleri group. European journal of clinical microbiology \&t infectious diseases : official publication of the European Society of Clinical Microbiology 2011;30:527-32. doi:10.1007/s10096010-1114-9

20. Centor RM. When should patients seek care for sore throat? Ann Intern Med 2013;159:636-7. doi:10.7326/00034819-159-9-201311050-00012

21. Little P, Watson L, Morgan S, Williamson I. Antibiotic prescribing and admissions with major suppurative complications of respiratory tract infections: a data linkage study. The British journal of general practice: the journal of the Royal College of General Practitioners 2002;52:187-90, 93.

22. Majeed A, Williams S, Jarman B, Aylin P. Prescribing of antibiotics and admissions for respiratory tract infections in England. Bmj 2004;329:879. doi:10.1136/bmj.329.7471.879

23. Sharland $M$, Kendall $H$, Yeates $D$, Randall A, Hughes G, Glasziou P, et al. Antibiotic prescribing in general practice and hospital admissions for peritonsillar abscess, mastoiditis, and rheumatic fever in children: time trend analysis. Bmj 2005;331:328-9. doi:10.1136/bmj.38503.706887.AE

24. Petersen I, Johnson AM, Islam A, Duckworth G, Livermore DM, Hayward AC. Protective effect of antibiotics against serious complications of common respiratory tract infections: retrospective cohort study with the UK General Practice Research Database. Bmj 2007;335:982. doi:10.1136/ bmj.39345.405243.BE

25. Little P, Stuart B, Hobbs FD, Butler CC, Hay AD, Campbell J, et al. Predictors of suppurative complications for acute sore throat in primary care: prospective clinical cohort study. Bmj 2013:347:f6867. doi:10.1136/bmj.f6867

26. Caballero M, Sabater F, Traserra J, Alos L, Bernal-Sprekelsen M. Epiglottitis and necrotizing fasciitis: a life-threatening complication of infectious mononucleosis. Acta oto-laryngologica 2005;125:1130-3. doi:10.1080/00016480510027475

27. Centor RM, Witherspoon JM, Dalton HP, Brody CE, Link K. The diagnosis of strep throat in adults in the emergency room. Medical decision making: an international journal of the Society for Medical Decision Making 1981;1:239-46. doi:10.1177/0272989X8100100304

28. Respiratory Tract Infections - Antibiotic Prescribing: Prescribing of Antibiotics for Self-Limiting Respiratory Tract Infections in Adults and Children in Primary Care. National Institute for Health and Clinical Excellence: Guidance London 2008.

29. Llor C, Cots JM, Bjerrum L, Cid M, Guerra G, Arranz X, et al. [Antibiotic prescribing in respiratory tract infections and predictive factors for their use]. Atencion primaria / Sociedad Española de Medicina de Familia y Comunitaria 2010;42:28-35.

30. Brien JH, Bass JW. Streptococcal pharyngitis: optimal site for throat culture. J Pediatr 1985;106:781-3. doi:10.1016/S0022-3476(85)80354-1

31. Kaplan EL, Gastanaduy AS, Huwe BB. The role of the carrier in treatment failures after antibiotic for group A streptococci in the upper respiratory tract. J Lab Clin Med 1981;98:326-35.

32. Llor C, Madurell J, Balague-Corbella M, Gómez M, Cots JM. Impact on antibiotic prescription of rapid antigen detection testing in acute pharyngitis in adults: a randomised clinical trial. The British journal of general practice: the journal of the Royal College of General Practitioners 2011;61:e244-51.

33. Dingle TC, Abbott AN, Fang FC. Reflexive culture in adolescents and adults with group a streptococcal pharyngitis. Clin Infect Dis 2014;59:643-50. doi:10.1093/cid/ ciu 400

34. Laxminarayan R, Duse A, Wattal C, Zaidi AK, Wertheim HF, Sumpradit N, et al. Antibiotic resistance-the need for global solutions. The Lancet Infectious diseases 2013;13:1057-98. doi:10.1016/S1473-3099(13)70318-9

35. Little P, Stuart B, Hobbs FD, Butler CC, Hay AD, Delaney B, et al. Antibiotic prescription strategies for acute sore throat: a prospective observational cohort study. The Lancet Infectious diseases 2014;14:213-9. doi:10.1016/ S1473-3099(13)70294-9

36. Giráldez-García C, Rubio B, Gallegos-Braun JF, Imaz I, González-Enríquez J, Sarria-Santamera A. Diagnosis and management of acute pharyngitis in a paediatric population: a cost-effectiveness analysis. European journal of pediatrics 2011;170:105967. doi:10.1007/s00431-011-1410-0

37. Cots JM MJ, Arranz J, Gómez M, Mórato ML, Sánchez C (editores). Manual de enfermedades infecciosas en Atención Primaria $3^{\text {a }}$ ed. Barcelona: semFYC Ediciones 2010.

38. Spinks A, Glasziou PP, Del Mar CB. Antibiotics for sore throat. Cochrane Database Syst Rev 2013;11:CD000023.

39. Zwart S, Sachs AP, Ruijs GJ, Gubbels JW, Hoes AW, de Melker RA. Penicillin for acute sore throat: randomised double blind trial of seven days versus three days treatment or placebo in adults. Bmj 2000;320:150-4. doi:10.1136/bmj.320.7228.150

40. Andrews M, Condren M. Once-daily amoxicillin for pharyngitis. J Pediatr Pharmacol Ther 2010;15:244-8.

41. Frye R, Bailey J, Blevins AE. Clinical inquiries. Which treatments provide the most relief for pharyngitis pain? J Fam Pract 2011;60:293-4.

42. Watson N, Nimmo WS, Christian J, Charlesworth A, Speight J, Miller K. Relief of sore throat with the anti-inflammatory throat lozenge flurbiprofen $8.75 \mathrm{mg}$ : a randomised, double-blind, placebo-controlled study of efficacy and safety. International journal of clinical practice 2000;54:490-6. 
43. Russo M, Bloch M, de Looze F, Morris C, Shephard A. Flurbiprofen microgranules for relief of sore throat: a randomised, double-blind trial. The British journal of general practice : the journal of the Royal College of General Practitioners 2013; 63:e149-55.

44. Chenot JF, Weber P, Friede T. Efficacy of Ambroxol lozenges for pharyngitis: a meta-analysis. BMC Fam Pract 2014;15:45. doi:10.1186/1471-229615-45

45. Ripoll MA. [Antimicrobials in primary care. Scenario and identification of problems]. Rev Esp Quimioter 2003;16:91-4.

46. Mir N, Trilla A, Quinto L, Molinero $\mathrm{M}$, Asenjo M. [Is there a place for otorhinolaryngology in primary care? Analysis of different areas].
Acta otorrinolaringologica espanola 2002;53:495-501. doi:10.1016/S00016519(02)78341-6

47. Asensio Nieto C. En: GSK e. Criterios de derivación ante patología ORL. España 2010:8-16.

48. Sánchez Gómez S. Otorrinolaringología en Atención Primaria Guía Práctica para el manejo de los procesos otorrinolaringológicos. España 2012:147-58.

49. Seguí Moya M, Pérez Fernández CA. Complicaciones de las infecciones orales y faríngeas Libro virtual de formación en ORL. España 2008;1-18.

50. Cervera Escario J, Del Castillo Martin F, Gómez Campdera JA, Gras Albert JR, Pérez Pinero B, Villafruela Sanz MA. [Indications for tonsillectomy and adenoidectomy: consensus document by the Spanish Society of
ORL and the Spanish Society of Pediatrics]. Acta otorrinolaringologica espanola 2006;57:59-65. doi:10.1016/ S0001-6519(06)78665-4

51. Guia d'actuació farmacèutica en el mal de gola. Consell de Col-legis Farmacèutics de Catalunya Barcelona 2014.

52. Foro de Atención Farmacéutica. Farmacia Comunitaria. Foro de Atención Farmacéutica. Farmacia Comunitaria. Guía Práctica para los Servicios de Atención Farmacéutica en la Farmacia Comunitaria. Madrid. 2010.

53. Bonafonte Jimeno MA Boleda Relats X, Ricote Belinchón M. Utilidad del Streptotest en la farmacia comunitaria para la discriminación rápida de faringitis bacteriana y vírica en pacientes adultos. Farmacéuticos Comunitarios 2013;5:59-63. 